\title{
Estimation of Azimuth Phase Undulations With Multisquint Processing in Airborne Interferometric SAR Images
}

\author{
Pau Prats and Jordi J. Mallorqui, Member, IEEE
}

\begin{abstract}
This letter presents a technique to detect and correct phase errors appearing in interferometric airborne synthetic aperture radar (SAR) systems due to the lack of precision in the navigation system. The technique is based on a multisquint processing approach, i.e., by processing the same image pairs with different squint angles we can combine the information of different interferograms to obtain the desired phase correction. Airborne single-pass interferometric data from the Deutsches Zentrum für Luft- und Raumfahrt (DLR) Experimental airborne SAR is used to validate the method.
\end{abstract}

Index Terms-Calibration, interferometry, processing, synthetic aperture radar (SAR).

\section{INTRODUCTION}

C URRENT airborne synthetic aperture radar (SAR) systems record the platform movement to later carry out the motion compensation during the processing. However, the performance of such systems is not enough to meet the requirements of subcentimetric precision. This lack of precision can lead to residual phase errors when obtaining the interferogram, inducing therefore important height errors in the final digital elevation model (DEM). Normally, these residual phase errors appear in the interferogram as low-frequency phase modulations in azimuth, i.e., phase undulations.

We present a technique able to detect and correct these undulations. The method is based on the processing of the same raw data with different squint angles. This approach can only be applied to SAR systems characterized by a relatively wide antenna beamwidth, as is the case of the Deutsches Zentrum für Luftund Raumfahrt (DLR) Experimental airborne SAR (E-SAR), which allows strong attitude variations during the data take with no impact on the final processed image. However, a second option for systems with a small beamwidth based on spectral diversity [1] is also feasible, where both interferograms are generated

Manuscript received February 14, 2003; revised April 15, 2003. This work was supported by the Spanish Commission for Science and Technology (CICYT) under Grant TIC2002-04451-C02-01 and by the Catalan Commission for Research (CIRIT).

P. Prats is with the Department of Signal Theory and Communications, Universitat Politècnica de Catalunya (UPC), E-08034, Spain, and is also with the Department of Telecommunications and Engineering Systems, Universitat Autònoma de Barcelona (UAB), Barcelona E-08193, Spain (e-mail: pprats@tsc.upc.es).

J. J. Mallorqui is with the Department of Signal Theory and Communications, Universitat Politècnica de Catalunya (UPC), Catalunya E-08034, Spain (e-mail: mallorqui@tsc.upc.es).

Digital Object Identifier 10.1109/TGRS.2003.814140

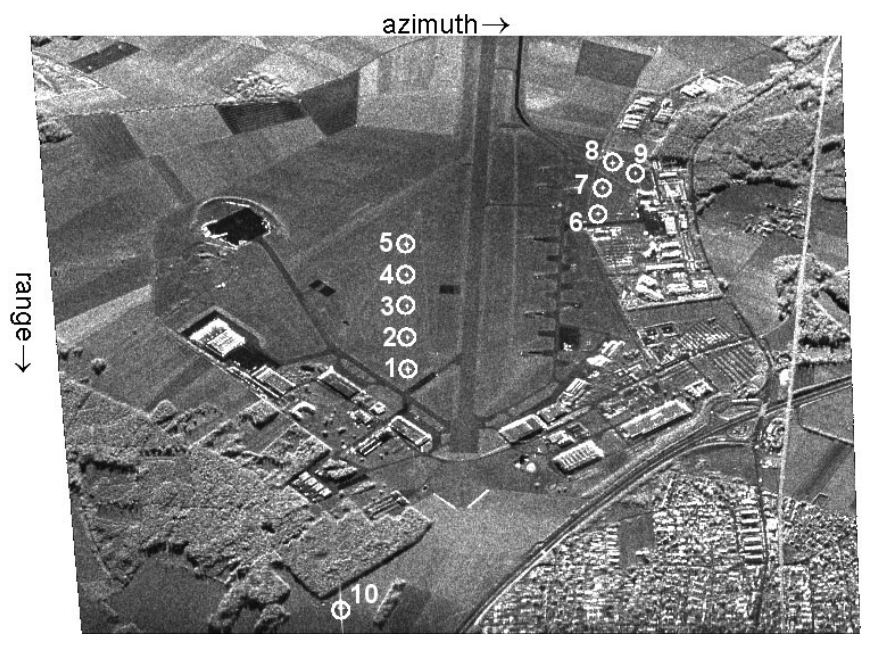

Fig. 1. Reflectivity image of the Oberpfaffenhofen test site with the position of all ten CRs marked and numbered in white (image in zero-Doppler geometry, processing squint $=3^{\circ}$ ).

from two subbands of the same processed image. The technique can be applied to both single- and repeat-pass systems.

To validate the proposed method with any loss of generality, airborne single-pass DLR's E-SAR data will be used. The data correspond to the test site of Oberpfaffenhofen, Germany, acquired in May 1998. The measurements were made in X-band (9.6 GHz) with a bandwidth of $100 \mathrm{MHz}$ and a pulse repetition frequency (PRF) of $1000 \mathrm{~Hz}$. The flight altitude is $3000 \mathrm{~m}$, and the velocity of the platform is $82 \mathrm{~m} / \mathrm{s}$. The radar look angle varies from $30^{\circ}$ up to $60^{\circ}$. Moreover, the test site contains ten corner reflectors (CRs) deployed on the ground, whose positions are perfectly known. As they are spread over different azimuth and range positions, they provide an excellent tool in order to verify the validity of the method. Fig. 1 shows the reflectivity image of the considered area, with the ten CRs highlighted and numbered in white.

A similar technique was presented in [2], which used the spectral diversity technique [1] to compute the azimuth registration error to later obtain the phase corrections. In [3], the phase undulations were detected, although they did not use that information to later compute the phase correction.

Section II presents the technique together with key issues concerning the processing of the images. In Section III, we present the experimental results, and finally, in Section IV, the conclusions. 


\section{MultisQuint PROCESSING}

When processing raw data of systems with a wide antenna beamwidth, a presumming step (i.e., filtering and subsampling [4]), typically of factor four, is carried out to reduce the azimuth bandwidth. Thus, it is possible to select the azimuth band to be processed (i.e., the Doppler centroid) within a relatively wide margin. In fact, we can choose any squint, taking into account that if we choose one different from the mean squint angle of the data take, the SNR will decrease and there may appear ghost responses in the image. This is important to allow combining images processed with different squints. For instance, with the validation data, the raw data lie approximately between the intervals of $(-310 \mathrm{~Hz}, 690 \mathrm{~Hz})$ at near range and $(-180 \mathrm{~Hz}, 820$ $\mathrm{Hz})$ at far range. This allows to perform a constant-squint processing with a bandwidth of $\mathrm{Baz}=250 \mathrm{~Hz}$ centered on the frequencies between $(150 \mathrm{~Hz}, 400 \mathrm{~Hz})$ - squint of $3^{\circ}$-at any point of the swath, maintaining still reasonable SNR and ambiguity suppression.

\section{A. Estimation of the Phase Undulations Using CRs}

The data have been processed with different squint angles, ranging from $-1^{\circ}$ to $8^{\circ}$ (note that a different squint angle means a certain pixel is being seen from different track positions). The computed phase in all ten corner reflectors has been corrected using the suggested method in [5] and [6], which modifies the azimuth compression filter to cancel the phase ramp in range for a given squint (normally equal to the processing squint). After the correction, the resulting phase errors (defined as the computed interferometric phase minus the theoretical one) in all ten corner reflectors are shown in Fig. 2, where the $x$ axis represents the azimuth position from where the corner was seen by the platform, being zero the center of the track. It must be noted that processing with a squint angle different from that of the data take reduces the SNR, but for the case of CRs the response is still strong enough to provide a phase with good quality.

Several comments can be made about Fig. 2. First of all, we can note a linear error along range, typical in airborne platforms due to inaccuracies in the calibration of the system. The correction of this error will not be treated here, as it is well explained in other sources [7], [8]. The second observation is the phase undulation. If the navigation data had no errors and the processor were well tuned, all CRs should show no phase variations along azimuth. However, Fig. 2 does show the presence of these phase azimuth undulations. One can note that all the corners have a similar behavior along azimuth (same local derivative).

A first approach would be to compute the local derivative of Fig. 2 for each CR. This way, we can average the derivatives of different CRs that correspond with the same along track position. After integrating we get an estimation of the phase undulations in azimuth. As we are computing the local derivative, it is not necessary to know the absolute phase of the CR.

\section{B. Estimation of Phase Undulations Without CRs}

Although the solution expounded before can correct these phase undulations, it has several disadvantages. In first place, there should be several CRs spread along the image and in different azimuth positions, and second, we should process the

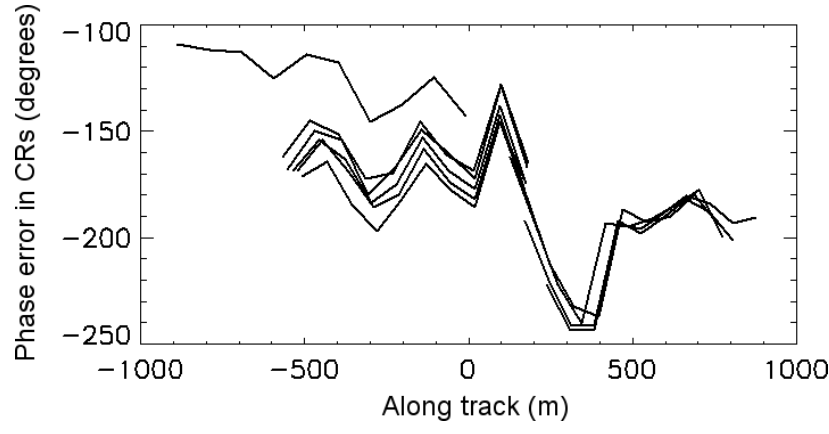

Fig. 2. Phase error of all ten CRs versus azimuth position.

image with several squints, a fact that, though feasible, not very efficient.

So, instead of using CRs, we can use highly coherent pixels from the interferogram, which do not have to necessarily contain CRs. The solution consists in the generation of a differential interferogram in order to compare the phase values of the different squint processed interferograms (we could, for example, subtract an interferogram processed with a squint of two to an interferogram processed with a squint of four). The phase of each pixel represents the phase difference, related to the derivative of the phase undulations. Furthermore, each pixel has been observed from different azimuth track positions depending on the selected processing squint. If we compute the differential phase and divide each range line by the distance between both geometries, we will obtain the derivative along azimuth. Therefore, after integrating the phase of this differential interferogram, we will obtain the desired phase correction in the mid point of the track between both geometries.

\section{Implementation Issues}

To better understand this technique, an analytical approach is preferred. We are considering azimuth compression is carried out by means of a matched filter tuned for every range bin. With this assumption, the interferogram phase is given by

$\phi=-\frac{4 \pi}{\lambda}\left(r_{1}-r_{2}\right)+2 \pi f_{C} \Delta t-2 \pi f_{0} \cdot\left(1-\cos \left(\beta_{\mathrm{eff}}\right)\right) \cdot \Delta \tau$

where $f_{c}$ is the Doppler-centroid; $\Delta t$ is the registration error in azimuth; $f_{0}$ is the carrier frequency; $\beta_{\text {eff }}$ is the effective squint angle as defined in [5]; and $\Delta \tau$ is the registration error in range. The first term in (1) contains the useful information, and the second and third ones are phase bias due to registration errors corresponding to the phase ramps in both azimuth (second term) and range (third term) dimensions. Note that we have not used the modified compression filter commented in [5] and [6] because in that case the first term would not be cancelled when generating the differential interferogram, as it would be multiplied by a different factor.

In the case of a single-pass system, although both channels can be coregistered in azimuth in a precise way, as the accuracy in the measurement of the position of both antennas is not perfect, we are having a residual motion error that induces a final registration error in azimuth, which causes the phase undulations. 
After obtaining two interferograms processed with different squints (e.g., four and two), we must adjust them to the same zero-Doppler geometry. Afterward, if we subtract their phases (i.e., we are generating a differential interferogram) we obtain

$$
\begin{aligned}
\phi_{\text {diff }} & (x, r) \\
= & 2 \pi \cdot f_{C A} \cdot \Delta t_{A}(x, r)-2 \pi \cdot f_{C B} \cdot \Delta t_{B}(x, r) \\
& -2 \pi f_{0} \cdot\left(1-\cos \left(\beta_{\mathrm{eff} A}(x, r)\right)\right) \cdot \Delta \tau_{A}(x, r) \\
& +2 \pi f_{0} \cdot\left(1-\cos \left(\beta_{\mathrm{eff} B}(x, r)\right)\right) \cdot \Delta \tau_{B}(x, r)
\end{aligned}
$$

where $x$ and $r$ are the azimuth and slant range positions, respectively; the subscripts $A$ and $B$ refer to the corresponding interferogram; and we have considered the registration error might be different in each interferogram. We are only interested in the first two terms, but we still have a phase bias due to a different phase ramp in range in both interferograms (third and fourth terms). However, it turns out that for low squint angles and small registration errors in range, these last two terms are small compared to the first two and, therefore they can be discarded. For the case of using spectral diversity, the phase ramp in range is the same and thus these phase bias are cancelled. Taking this into account, the differential phase for one given pixel can be rewritten as

$$
\begin{aligned}
\phi_{\text {diff }}\left(\frac{x_{A}+x_{B}}{2} ; x_{0}, r_{0}\right) \\
\quad=\phi_{A}\left(x_{A} ; x_{0}, r_{0}\right)-\phi_{B}\left(x_{B} ; x_{0}, r_{0}\right)
\end{aligned}
$$

where $\phi_{A}$ and $\phi_{B}$ correspond to the phase error of each interferogram related with the registration error in azimuth, and $x_{0}$, $r_{0}$ represent the position of the pixel in the image. The values $x_{A}$ and $x_{B}$ correspond to the beam-center position of the track for each processed squint. Each differential phase value is assigned to the mid position between both geometries, where the correction will be applied.

Averaging is required because the obtained differential phase is very noisy. The best approach is to average the normalized complex samples masking non valid points by means of a coherence threshold. However, as a first step, it is recommended to convert the differential interferogram to a beam-center geometry as the phase differences, for a given azimuth position, are almost constant throughout range in this geometry. This should be carried out by using the mean squint angle between both processed squints $\beta_{A}$ and $\beta_{B}$. A large multilook factor in the range dimension is preferred. To finally obtain the phase derivative we divide the phase by the distance between both acquisitions geometries given by

$$
\Delta x(r)=\left(\tan \left(\beta_{A}\right)-\tan \left(\beta_{B}\right)\right) \cdot r
$$

where $\beta_{A}$ is the large squint and $\beta_{B}$ the small one. Afterward, we have just to integrate to obtain the phase azimuth undulations

$$
\phi_{\text {undul }}(x, r)=\int \frac{\phi_{\text {diff }}(x, r)}{\Delta x(r)} d x+C
$$

where $\phi_{\text {diff }}$ is the phase of the differential interferogram; $\phi_{\text {undul }}$ is the computed phase undulations; and $C$ is an unknown phase offset for the whole image. Note that the CRs do not influence in

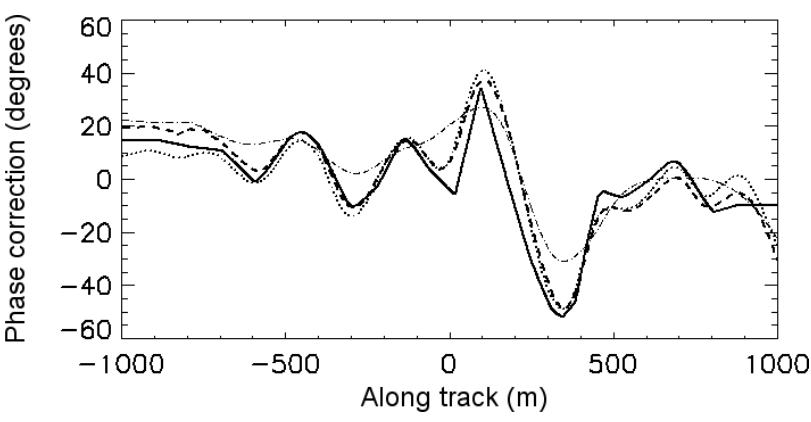

Fig. 3. Estimated phase undulations for midrange computed with CRs (solid), the proposed method using squints 4 and 3 (dashed), the proposed method using squints 5 and 2 (dashed-dotted), and the proposed method with spectral diversity of a $3^{\circ}$ squint processed image (dotted)

the results as they just represent a few pixels in the single-look complex (SLC).

\section{Algorithm Accuracy}

The algorithm accuracy can be estimated starting from the well-known formula for the standard deviation of the phase when the number of looks is equal or greater than four [9]

$$
\sigma_{\phi}=\sqrt{\frac{1}{2 \cdot N_{L}} \frac{1-|\gamma|^{2}}{|\gamma|^{2}}}
$$

where $N_{L}$ is the number of looks, and $\gamma$ is the coherence. Assuming both processed interferograms are statistically independent (this can be assumed only when there is no overlapped spectra between both interferograms), the standard deviation of the differential interferogram becomes

$$
\sigma_{\phi_{\text {diff }}}=\sqrt{2} \cdot \sigma_{\phi}
$$

and the standard deviation of the derivative

$$
\sigma_{\phi_{\text {deriv }}}(r)=\frac{\sigma_{\phi_{\text {diff }}}}{\Delta x(r)} .
$$

That is to say, the further the separation between processed squints, the lower the noise in the differential interferogram, and the better the performance of the algorithm. It must be noted that if the separation is too large, we might not detect rapid variations, and also we might obtain a noisy wrapped phase in (3), which would be quite difficult to unwrap. In the next section we will show the importance of this fact.

\section{EXPERIMENTAL RESULTS}

The proposed technique has been validated with data from the E-SAR. Fig. 3 shows the computed correction for midswath using the CRs (solid), the proposed method with squints 4 and 3 (dashed), the proposed method using squints 5 and 2 (dashed-dotted), and the proposed method using spectral diversity (dotted). In the last three cases, a multilook window of $20 \times 80$ (azimuth $\times$ range) has been used with a coherence threshold of 0.8 . As already commented, the large multilook window in range can be applied as the phase differences occur along azimuth and depend only slightly on the range dimension in a beam-center geometry. 


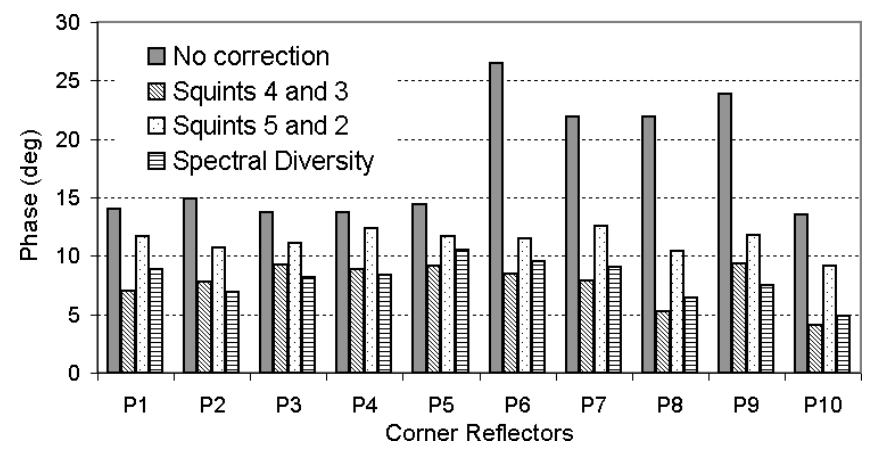

Fig. 4. Standard deviation of the phase error for each CR before and after having applied the corresponding corrections.
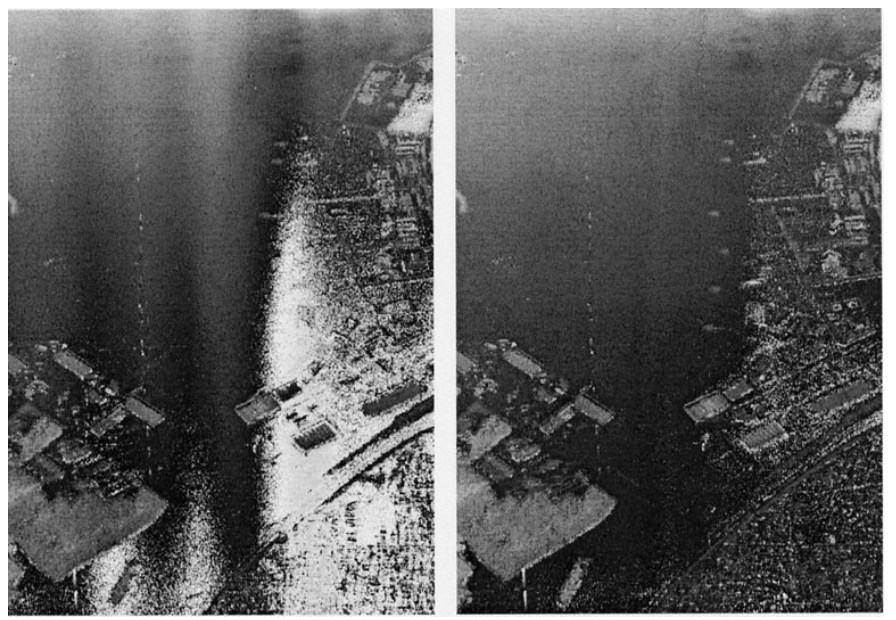

Fig. 5. Detail of the original phase of the interferogram where the azimuth undulations are (left) noticeable and (right) detail of phase corrected with the proposed method using squints 4 and 3 .

As it can be noted in Fig. 3, the proposed method matches quite accurately the curve obtained with the CRs. The standard deviation of the phase errors for each $\mathrm{CR}$ for all processed squints (from -1 to 8 ) after having applied the three corrections appears in Fig. 4. For squints 4 and 3 and with spectral diversity [1], we can see how their phase error along azimuth has been reduced. In Fig. 5, we can observe a detail of the phase before and after the correction. The technique has been able to correctly compute and reduce the phase azimuth undulations. Note that with the system configuration of the E-SAR in X-band, an error of $50^{\circ}$ translates into height errors of 3-7 m depending on the radar look angle.

In Fig. 3, we can see how the correction computed using squints 5 and 2 does not follow the variations of the undulations fast enough, and therefore, in this case a smaller separation is preferred. In principle, there is no prior way to know how fast the undulations will be, so a small separation should be chosen, with the option to increase it to reduce the noise. Results have shown that a separation between both processed interferograms of approximately $1^{\circ}$, in combination with a large multilook factor in range, can detect in a satisfactory way the undulations.

\section{CONCLUSION}

A new technique for the estimation of azimuth phase undulations without the need of CRs has been successfully validated, allowing the obtaining of better quality DEMs. This method can detect and correct phase undulations appearing in interferograms due to uncompensated motion errors in systems with a wide antenna beamwidth by combining two full-resolution interferograms processed with different squints. In the case of systems with a smaller azimuth antenna beamwidth, each SLC can be split to generate two low-resolution interferograms and afterward apply the algorithm.

\section{ACKNOWLEDGMENT}

The authors wish to thank M. Bara for useful discussions and to the Deutsches Zentrum für Luft- und Raumfahrt (DLR) for supplying the E-SAR data. They would also like to thank the anonymous reviewers for their comments and suggestions.

\section{REFERENCES}

[1] R. Scheiber and A. Moreira, "Coregistration of interferometric SAR images using spectral diversity," IEEE Trans. Geosci. Remote Sensing, vol. 38, pp. 2179-2191, Sept. 2000.

[2] A. Reigber, "Correction of residual motion errors in airborne SAR interferometry," IEE Electron. Lett., vol. 37, no. 17, pp. 1083-1084, Aug. 2001.

[3] M. Bara, J. Andreu, R. Scheiber, A. Moreira, and A. Broquetas, "Interferometric phase corrections during squinted-data geocoding," in Proc. EUSAR, Munich, Germany, May 2000.

[4] D. Massonnet, F. Adragna, and M. Rossi, "CNES general-purpose SAR correlator," IEEE Trans. Geosci. Remote Sensing, vol. 32, pp. 636-643, May 1994.

[5] M. Bara, R. Scheiber, A. Broquetas, and A. Moreira, "Interferometric SAR signal analysis in the presence of squint," IEEE Trans. Geosci. Remote Sensing, vol. 5, pp. 2164-2178, Sept. 2000.

[6] M. Bara, P. Prats, J. J. Mallorqui, and J. Lopez, "Validation of SAR interferometric phase corrections in the squinted case for DEM quality enhancement," IEE Electron. Lett., vol. 38, no. 22, pp. 1380-1381, Oct. 2002.

[7] M. Bara, "Airborne SAR interferometric techniques for mapping applications," Ph.D. dissertation, Dept. Electromagnetics and Photonics Group, Univ. Politècnica de Catalunya, Barcelona, Spain, 2000.

[8] J. J. Mallorqui, I. Rosado, and M. Bara, "Interferometric calibration for DEM enhancing and system characterization in single pass SAR interferometry," in Proc. IGARSS, 2001, pp. 404-406.

[9] E. Rodriguez and J. M. Martin, "Theory and design of interferometric synthetic aperture radars," Proc. Inst. Elect. Eng. F, vol. 139, no. 2, pp. 147-159, Apr. 1992. 